\title{
Single-logarithmic stability for the Calderón problem with local data *
}

\author{
Giovanni Alessandrini, ${ }^{\dagger}$ Kyoungsun Kim ${ }^{\ddagger}$
}

\begin{abstract}
We prove an optimal stability estimate for Electrical Impedance Tomography with local data, in the case when the conductivity is precisely known on a neighborhood of the boundary. The main novelty here is that we provide a rather general method which enables to obtain the Hölder dependence of a global Dirichlet to Neumann map from a local one on a larger domain when, in the layer between the two boundaries, the coefficient is known.
\end{abstract}

\section{Introduction}

In this paper we consider the stability issue for Electrical Impedance Tomography (or, as is the same, the Calderón problem) in the case of local boundary data. As a consequence of the recent results of uniqueness [7, 13, 14, 15, there has also been a number of results of stability [11, 12, 9], either directly associated to the conductivity equation setting, or associated to the Schrödinger equation setting. In general, the problem of the optimal rate of stability it is not yet settled.

It is expected however that, if the unknown coefficient (either the conductivity or the potential) is a-priori given on a neighborhood of the boundary then the rate of stability is of logarithmic type with a single logarithm, which is optimal, in view of the well-known examples by Mandache [18]. This is in fact the result of Fathallah [9], which along the lines of previous uniqueness results by Lassas, Cheney and Uhlmann [16] and Ammari and Uhlmann [6], proves a stability result with a single logarithm in the setting of the Schrödinger equation.

Here we consider the same situation directly for the conductivity equation, see Theorem 2.5 in the next section, with the principal aim of providing a method of proof which might be adapted to other inverse boundary problems with local data

\footnotetext{
${ }^{*}$ Work supported in part by PRIN 20089PWTPS and by the JSPS International Training Program (ITP).

${ }^{\dagger}$ Dipartimento di Matematica e Geoscienze, Università degli Studi di Trieste, via Valerio 12/1, 34127 Trieste, Italy. E-mail: alessang@units.it

${ }^{\ddagger}$ Department of Mathematics, Inha University, Incheon 402-751, Korea. E-mail: kskim@inha.ac.kr
} 
and in which the unknown parameters, or objects (such as cavities, inclusions or cracks) are a-priori known to be located at a positive distance from the boundary.

The main idea in this method is that, assuming that the unknown part of a coefficient $\gamma$ is concentrated in a subset $D \subset \subset \Omega$, and if we fix a bigger domain $\widetilde{D}$ such that $D \subset \subset \widetilde{D} \subset \subset \Omega$, then the full Dirichlet to Neumann map $\Lambda_{\gamma}^{\partial \widetilde{D}}$ associated to $\widetilde{D}$ can be determined by the local Dirichlet to Neumann map $\Lambda_{\gamma}^{\Sigma}$ associated to a portion $\Sigma$ of $\partial \Omega$. In fact such a dependence has a Hölder rate of stability. This is the essence of Theorem 3.1 below.

In Section 2 we formulate that main assumptions and state the main Theorem 2.5. Section 3 starts with some geometrical construction needed for the precise formulation of Theorem 3.1 which, in combination with the standard stability estimate with the full Dirichlet to Neumann map Theorem 3.2, enables a proof of Theorem 2.5. The final Section 4 is devoted to the proof of Theorem 3.1, this is mainly based on the use of singular solutions and on estimates of propagation of smallness for solutions of elliptic equations, some ideas are borrowed from previous work in [3, 5, 8].

\section{Notation and main assumptions}

Let us introduce some notation and definitions.

Given $x \in \mathbb{R}^{n}, n \geq 3$, we shall denote $x=\left(x^{\prime}, x_{n}\right)$, where $x^{\prime}=\left(x_{1}, \ldots, x_{n-1}\right) \in$ $\mathbb{R}^{n-1}, x_{n} \in \mathbb{R}$. Given $x \in \mathbb{R}^{n}, r>0$, we shall use the following notation for balls and cylinders.

$$
\begin{gathered}
B_{r}(x)=\left\{y \in \mathbb{R}^{n}|| y-x \mid<r\right\}, \quad B_{r}=B_{r}(0), \\
B_{r}^{\prime}\left(x^{\prime}\right)=\left\{y^{\prime} \in \mathbb{R}^{n-1}|| y^{\prime}-x^{\prime} \mid<r\right\}, \quad B_{r}^{\prime}=B_{r}^{\prime}(0), \\
\Gamma_{a, b}(x)=\left\{y=\left(y^{\prime}, y_{n}\right) \in \mathbb{R}^{n}|| y^{\prime}-x^{\prime}|<a,| y_{n}-x_{n} \mid<b\right\}, \quad \Gamma_{a, b}=\Gamma_{a, b}(0) .
\end{gathered}
$$

We shall denote by $\Omega$ a bounded open connected subset of $\mathbb{R}^{n}$. We shall assume that its boundary is Lipschitz according to the following definition.

Definition 2.1 We say that the boundary of $\Omega$ is of Lipschitz class with constants $\rho_{0}, M_{0}>0$, if, for any $P \in \partial \Omega$, there exists a rigid transformation of coordinates under which $P=0$ and

$$
\Omega \cap \Gamma_{\frac{\rho_{0}}{M_{0}}, \rho_{0}}(P)=\left\{x=\left(x^{\prime}, x_{n}\right) \in \Gamma_{\frac{\rho_{0}}{M_{0}}, \rho_{0}} \quad \mid \quad x_{n}>Z\left(x^{\prime}\right)\right\},
$$

where $Z: B_{\frac{\rho_{0}}{M_{0}}}^{\prime} \rightarrow \mathbb{R}$ is a Lipschitz function satisfying

$$
\begin{gathered}
Z(0)=0, \\
\|Z\|_{C^{0,1}\left(B_{\frac{\rho_{0}}{M_{0}}}^{\prime}\right)} \leq M_{0} \rho_{0} .
\end{gathered}
$$


We shall consider an open subset $\Sigma$ of $\partial \Omega$ which is accessible to measurements. We shall require a lower bound on its smallness as follows.

Definition 2.2 We shall say that $\Sigma$ has size at least $d_{0}, 0<d_{0} \leq \rho_{0}$, if there exists at least one point $P \in \Sigma$ such that

$$
\operatorname{dist}(P, \partial \Omega \backslash \Sigma) \geq d_{0} .
$$

Consider a conductivity coefficient $\gamma$ defined in $\Omega$ and let us assume it is a bounded measurable function which satisfies the ellipticity condition

$$
\lambda<\gamma(x)<\lambda^{-1} \quad \text { for all } x \in \Omega,
$$

for a given positive constant $\lambda$.

Definition 2.3 We define the space of localized Dirichlet data as follows

$$
H_{c o}^{1 / 2}(\Sigma)=\left\{\varphi \in H^{1 / 2}(\partial \Omega) \mid \operatorname{supp} \varphi \subset \Sigma\right\}
$$

and we denote by $H_{c o}^{-1 / 2}(\Sigma)$ its topological dual space. We denote with $\langle\cdot, \cdot\rangle$ the dual pairing between these two spaces, based on the standard $L^{2}(\partial \Omega)$ inner product. We shall denote by $\|\cdot\|_{\mathcal{L}\left(H_{c o}^{1 / 2}(\Sigma), H_{c o}^{-1 / 2}(\Sigma)\right)}$ the operator norm on the space of bounded linear operators from $H_{c o}^{1 / 2}(\Sigma)$ into $H_{c o}^{-1 / 2}(\Sigma)$.

Now, for each $\varphi \in H_{c o}^{1 / 2}(\Sigma)$, consider the weak solution $u \in H^{1}(\Omega)$ to the Dirichlet problem

$$
\left\{\begin{array}{l}
\operatorname{div}(\gamma \nabla u)=0 \quad \text { in } \Omega \\
\left.u\right|_{\partial \Omega}=\varphi \text { on } \partial \Omega .
\end{array}\right.
$$

We introduce the local Dirichlet to Neumann map $\Lambda_{\gamma}^{\Sigma}$ as the map which associates to the Dirichlet data $\varphi \in H_{c o}^{1 / 2}(\Sigma)$ the boundary co-normal derivative $\left.\gamma \frac{\partial u}{\partial \nu}\right|_{\Sigma}$, where $\nu$ is the outward unit normal to $\partial \Omega$. More precisely, we introduce the following definition.

Definition 2.4 The map

$$
\Lambda_{\gamma}^{\Sigma}: H_{c o}^{1 / 2}(\Sigma) \rightarrow H_{c o}^{-1 / 2}(\Sigma)
$$

is the operator characterized by

$$
\left\langle\Lambda_{\gamma}^{\Sigma} \varphi, \psi\right\rangle=\int_{\Omega} \gamma \nabla u \cdot \nabla v, \quad \text { for every } \varphi, \psi \in H_{c o}^{1 / 2}(\Sigma)
$$

where $u$ is the solution to the Dirichlet problem (2.6) and $v$ is any function in $H^{1}(\Omega)$ such that $\left.v\right|_{\partial \Omega}=\psi$. 
We shall consider an open subset $D$ of $\Omega$ whose boundary is also Lipschitz with constants $\rho_{0}, M_{0}$ and which is at a given positive distance from $\partial \Omega$, namely we assume

$$
\operatorname{dist}(D, \partial \Omega) \geq \rho_{0} .
$$

On the unknown conductivity $\gamma$ we shall assume the following a-priori regularity bound

$$
\|\gamma\|_{W^{2, \infty}(\Omega)} \leq E
$$

and also that it is precisely known outside $D$. That is, we assume that we are given a reference conductivity $\gamma_{0}$ which satisfies (2.5) and (2.9) and the unknown $\gamma$ satisfies

$$
\gamma=\gamma_{0} \text { in } \Omega \backslash \bar{D}
$$

Theorem 2.5 Let $\Omega, \Sigma$ and $D$ satisfy the above stated assumptions. If $\gamma_{1}, \gamma_{2}$ satisfy (2.5), (2.9) and (2.10), then we have

$$
\left\|\gamma_{1}-\gamma_{2}\right\|_{L^{\infty}(\Omega)} \leq \omega\left(\left\|\Lambda_{\gamma_{1}}^{\Sigma}-\Lambda_{\gamma_{2}}^{\Sigma}\right\|_{\mathcal{L}\left(H_{c o}^{1 / 2}(\Sigma), H_{c o}^{-1 / 2}(\Sigma)\right)}\right)
$$

where $\omega(t)$ is an increasing function of $t \geq 0$ such that

$$
\omega(t) \leq C|\log t|^{-\delta} \text { for every } 0<t<e^{-1},
$$

here $C>0$ only depends on the a-priori data $\lambda, E, \rho_{0}, M_{0}, d_{0}, \operatorname{diam}(\Omega)$ and on $n$, whereas $\delta \in(0,1)$ only depends on $n$.

\section{Proof of Theorem 2.5}

Before formulating the main new tool (Theorem 3.1 below) that we shall use for the proof of Theorem 2.5, we need to introduce some geometrical constructions. We use notation and some results described in [4].

First we introduce an augmented domain $\widetilde{\Omega}$ by attaching to $\Omega$ an open set $\mathcal{A}$ lying in its exterior and whose boundary intersects $\partial \Omega$ on an open portion $\Sigma_{0} \subset \subset \Sigma$. We refer to [4, Section 6] for details. In particular, we can choose $\mathcal{A}$ in such a way that, setting $\widetilde{\Omega}=\Omega \cup \Sigma_{0} \cup \mathcal{A}$, the following properties hold.

There exist $\rho_{1}, M_{1}>0$, only depending on $\rho_{0}, M_{0}, d_{0}$, such that

(i) $\widetilde{\Omega}$ is open, connected and has Lipschitz boundary with constants $\rho_{1}, M_{1}>0$,

(ii) There exists $Q \in \mathcal{A}$ such that

$$
B_{2 \rho_{1}}(Q) \subset \mathcal{A}
$$


Next, if we denote, for any open set $E \in \mathbb{R}^{n}$ and $h>0$,

$$
E_{h}=\{x \in E \mid \operatorname{dist}(x, \partial E)>h\}
$$

we observe that there exists $h_{0}>0$ only depending on $\rho_{0}, M_{0}, d_{0}$ such that $\widetilde{\Omega}_{h}$ is connected for every $h \leq h_{0}$, see for instance [4, Proposition 5.5]. Note that $B_{\rho_{1}}(Q) \subset \widetilde{\Omega}_{h}$ if $h \leq \rho_{1}$.

We introduce two domains $D^{\prime}, \widetilde{D}$ nested as follows

$$
D \subset \subset D^{\prime} \subset \subset \widetilde{D} \subset \subset \Omega \text {. }
$$

Such domains can be chosen in such a way that for suitable $\rho_{2}, M_{2}>0$, only depending on $\rho_{0}, M_{0}, d_{0}$, we have

(i) $\widetilde{\Omega} \backslash \overline{D^{\prime}}$ and $\widetilde{\Omega} \backslash \overline{\widetilde{D}}$ are connected,

(ii) $D^{\prime}, \widetilde{D}$ have $C^{2}$ boundaries, satisfying a Lipschitz condition with constants $\rho_{2}, M_{2}$,

(iii) the boundaries of $D, D^{\prime}, \widetilde{D}, \widetilde{\Omega}_{\rho_{2}}$ have mutual distance greater than $\rho_{2}$.

Let us incidentally note that the set $\widetilde{D}$ shall be used right away in the following statement, Theorem 3.1, whereas the introduction of $D^{\prime}$ shall be justified during the proof of the same Theorem 3.1 .

Now we introduce the usual Dirichlet to Neumann map $\Lambda_{\gamma}^{\partial \widetilde{D}}$ associated to the domain $\widetilde{D}$. Namely, for a conductivity coefficient $\gamma$ satisfying (2.5), we consider, for any $\eta \in H^{1 / 2}(\partial \widetilde{D})$, the solution $v \in H^{1}(\widetilde{D})$ to the Dirichlet problem

$$
\left\{\begin{array}{l}
\operatorname{div}(\gamma \nabla v)=0 \text { in } \widetilde{D} \\
v=\eta \text { on } \partial \widetilde{D}
\end{array}\right.
$$

and we define

$$
\Lambda_{\gamma}^{\partial \widetilde{D}}(\eta)=\left.\gamma \frac{\partial v}{\partial \nu}\right|_{\partial \widetilde{D}}
$$

where $\nu$ is the outward unit normal to $\partial \widetilde{D}$. Again, $\Lambda_{\gamma}^{\partial \widetilde{D}}$ is identified through the bilinear form characterization

$$
\left\langle\Lambda_{\gamma}^{\partial \widetilde{D}} \eta, \xi\right\rangle=\int_{\widetilde{D}} \gamma \nabla v \cdot \nabla w, \quad \text { for every } \eta, \xi \in H^{1 / 2}(\partial \widetilde{D}),
$$

where $v$ is the solution to (3.1) and $w$ is any function in $H^{1}(\widetilde{D})$ such that $\left.w\right|_{\partial \widetilde{D}}=\xi$.

Theorem 3.1 Let $\Omega, \Sigma, D$ and $\widetilde{D}$ satisfy the above stated assumptions. If $\gamma_{1}, \gamma_{2}$ satisfy (2.5), (2.9) and (2.10), then we have

$$
\left\|\Lambda_{\gamma_{1}}^{\partial \widetilde{D}}-\Lambda_{\gamma_{2}}^{\partial \widetilde{D}}\right\|_{\mathcal{L}\left(H^{1 / 2}(\partial \widetilde{D}), H^{-1 / 2}(\partial \widetilde{D})\right)} \leq C\left\|\Lambda_{\gamma_{1}}^{\Sigma}-\Lambda_{\gamma_{2}}^{\Sigma}\right\|_{\mathcal{L}\left(H_{c o}^{1 / 2}(\Sigma), H_{c o}^{-1 / 2}(\Sigma)\right)}^{\beta},
$$

where $C>0, \beta \in(0,1)$ only depend on $\lambda, E, \rho_{0}, M_{0}, d_{0}$, $\operatorname{diam}(\Omega)$ and on $n$. 
The proof of this theorem is the content of the next section. The other main ingredient for the proof of Theorem 2.5] is the following known stability result for the Calderón problem with full boundary data, see [1] and also, for details, [2].

Theorem 3.2 Let $\widetilde{D}$ be as above. Suppose that $\gamma_{1}, \gamma_{2}$ satisfy (2.5) and (2.9). We have the following stability estimate

$$
\left\|\gamma_{1}-\gamma_{2}\right\|_{L^{\infty}(\widetilde{D})} \leq \omega\left(\left\|\Lambda_{\gamma_{1}}^{\partial \widetilde{D}}-\Lambda_{\gamma_{2}}^{\partial \widetilde{D}}\right\|_{\mathcal{L}\left(H^{1 / 2}(\partial \widetilde{D}), H^{-1 / 2}(\partial \tilde{D})\right)}\right)
$$

where $\omega$ is a logarithmic modulus of continuity satisfying

$$
\omega(t) \leq C|\log t|^{-\delta} \text { for every } 0<t<e^{-1},
$$

and $C>0$ only depends on the a-priori data $\lambda, E, \rho_{0}, M_{0}, d_{0}, \operatorname{diam}(\Omega)$ and on $n$, whereas $\delta \in(0,1)$ only depends on $n$.

Hence, assuming Theorem 3.1 proven, we can conclude as follows.

Proof of Theorem 2.5. Let us denote $\left\|\Lambda_{\gamma_{1}}^{\Sigma}-\Lambda_{\gamma_{2}}^{\Sigma}\right\|=\varepsilon$. By (3.4), we have

$$
\left\|\Lambda_{\gamma_{1}}^{\partial \widetilde{D}}-\Lambda_{\gamma_{2}}^{\partial \widetilde{D}}\right\|_{\mathcal{L}\left(H^{1 / 2}(\partial \widetilde{D}), H^{-1 / 2}(\partial \widetilde{D})\right)} \leq C \varepsilon^{\beta}
$$

where, without loss of generality we may assume $C \geq 1$. If, on one hand, we have $C \varepsilon^{\beta}<e^{-1}$ then by (3.5)

$$
\begin{array}{r}
\left\|\gamma_{1}-\gamma_{2}\right\|_{L^{\infty}(\Omega)}=\left\|\gamma_{1}-\gamma_{2}\right\|_{L^{\infty}(\widetilde{D})} \leq \\
\leq C\left|\log \left(C \varepsilon^{\beta}\right)\right|^{-\delta} \leq C\left(\frac{\log C e}{\beta}\right)^{\delta}|\log \varepsilon|^{-\delta} .
\end{array}
$$

On the other hand, if $C \varepsilon^{\beta} \geq e^{-1}$ then, trivially,

$$
\begin{array}{r}
\left\|\gamma_{1}-\gamma_{2}\right\|_{L^{\infty}(\Omega)} \leq \lambda^{-1} \leq \\
\leq \lambda^{-1}\left(\frac{\log C e}{\beta}\right)^{\delta}|\log \varepsilon|^{-\delta}
\end{array}
$$

and the thesis follows.

\section{Proof of Theorem 3.1}

Let us begin by observing that the reference conductivity $\gamma_{0}$ can be extended to $\widetilde{\Omega}$ in such a way that the ellipticity condition (2.5) continues to hold in all of $\widetilde{\Omega}$ and that the following Lipschitz bound holds

$$
\left\|\gamma_{0}\right\|_{W^{1, \infty}(\widetilde{\Omega})} \leq E_{1}
$$


where $E_{1}$ only depends on $E, \rho_{0}, M_{0}, d_{0}$. The same extension can be used for any $\gamma$ satisfying (2.10), and from now on we shall replace this assumption with the following one

$$
\gamma=\gamma_{0} \text { in } \widetilde{\Omega} \backslash \bar{D}
$$

Let $\gamma_{1}, \gamma_{2}$ be the two conductivities appearing in the statement of Theorem 3.1 , let us introduce the Green's function $G_{i}(x, y), i=1,2$, for the operator for $\operatorname{div}\left(\gamma_{i} \nabla\right)$ in the domain $\widetilde{\Omega}$, that is, for any $y \in \widetilde{\Omega}, G_{i}(\cdot, y)$ is defined as the distributional solution to

$$
\left\{\begin{array}{l}
\operatorname{div}_{x}\left(\gamma_{i}(\cdot) \nabla_{x} G_{i}(\cdot, y)\right)=-\delta(\cdot-y) \quad \text { in } \widetilde{\Omega}, \\
G_{i}(\cdot, y)=0 \quad \text { on } \partial \widetilde{\Omega} .
\end{array}\right.
$$

As is well-known, $G_{i}(x, y)$ is symmetric, it has a singularity on the diagonal $\{x=y\}$ of the order of $|x-y|^{2-n}$, and, away from the diagonal, it is $C^{1, \alpha}$-regular in each of the two variables $x, y$, moreover also the mixed derivatives $\nabla_{x} \nabla_{y} G_{i}(x, y)$ exist and are locally Hölder continuous away from the diagonal, see for instance [10, Theorem 8.32]. In particular we shall make use of the following energy bound.

Proposition 4.1 For every $y \in \widetilde{\Omega}$ and every $r>0$ we have

$$
\int_{\widetilde{\Omega} \backslash B_{r}(y)}\left|\nabla_{x} G_{i}(x, y)\right|^{2} d x \leq C r^{2-n},
$$

where $C>0$ only depends on $\lambda$ and $n$.

Proof. A proof can be easily obtained through a Caccioppoli type inequality and the well-known pointwise upper bound of the Green's function [17, details can be found in [5, Proposition 3.1].

Let us fix $\eta_{i} \in C^{1, \alpha}(\partial \widetilde{D}), i=1,2$ for some $\alpha \in(0,1)$ and consider $v_{i}$ be solutions to the Dirichlet problems

$$
\left\{\begin{array}{l}
\operatorname{div}\left(\gamma_{i} \nabla v_{i}\right)=0 \text { in } \widetilde{D} \\
v_{i}=\eta_{i} \text { on } \partial \widetilde{D}
\end{array}\right.
$$

By Green's formula, for every $x \in \widetilde{D}$, we obtain

$$
\begin{aligned}
v_{i}(x) & =\int_{\partial \widetilde{D}}\left[\gamma_{i}(z) \frac{\partial v_{i}}{\partial \nu}(z) G_{i}(x, z)-v_{i}(z) \gamma_{i}(z) \frac{\partial G_{i}}{\partial \nu_{z}}(x, z)\right] d \sigma_{z} \\
& =\int_{\partial \widetilde{D}} \gamma_{0}(z)\left[\frac{\partial v_{i}}{\partial \nu}(z) G_{i}(x, z)-v_{i}(z) \frac{\partial G_{i}}{\partial \nu_{z}}(x, z)\right] d \sigma_{z}
\end{aligned}
$$

Note that, by the regularity assumptions on the conductivities and on $\partial \widetilde{D}, v_{1}, v_{2}$ are differentiable, in the classical sense, up to the boundary of $\widetilde{D}$ and that differentiation 
under the integral is elementarily allowed in the above formulas. Therefore, by Fubini's theorem, for every $x \in D$

$$
\begin{aligned}
\nabla v_{1}(x) & \cdot \nabla v_{2}(x)= \\
= & \int_{\partial \widetilde{D} \times \partial \widetilde{D}} \gamma_{0}(z) \gamma_{0}(w) \frac{\partial v_{1}}{\partial \nu}(z) \frac{\partial v_{2}}{\partial \nu}(w) \nabla_{x} G_{1}(x, z) \cdot \nabla_{x} G_{2}(x, w) d \sigma_{z} \times \sigma_{w} \\
& -\int_{\partial \widetilde{D} \times \partial \widetilde{D}} \gamma_{0}(z) \gamma_{0}(w) \frac{\partial v_{1}}{\partial \nu}(z) v_{2}(w) \nabla_{x} G_{1}(x, z) \cdot \nabla_{x} \frac{\partial G_{2}}{\partial \nu_{w}}(x, w) d \sigma_{z} \times \sigma_{w} \\
& -\int_{\partial \widetilde{D} \times \partial \widetilde{D}} \gamma_{0}(z) \gamma_{0}(w) v_{1}(z) \frac{\partial v_{2}}{\partial \nu}(w) \nabla_{x} \frac{\partial G_{1}}{\partial \nu_{z}}(x, z) \cdot \nabla_{x} G_{2}(x, w) d \sigma_{z} \times \sigma_{w} \\
& +\int_{\partial \widetilde{D} \times \partial \widetilde{D}} \gamma_{0}(z) \gamma_{0}(w) v_{1}(z) v_{2}(w) \nabla_{x} \frac{\partial G_{1}}{\partial \nu_{z}}(x, z) \cdot \nabla_{x} \frac{\partial G_{2}}{\partial \nu_{w}}(x, w) d \sigma_{z} \times \sigma_{w}
\end{aligned}
$$

where $\sigma$ denotes the surface measure on $\partial \widetilde{D}$. For any $z, w \in \widetilde{\Omega} \backslash \bar{D}$ let us define

$$
S(z, w)=\int_{D}\left(\gamma_{1}(x)-\gamma_{2}(x)\right) \nabla_{x} G_{1}(x, z) \cdot \nabla_{x} G_{2}(x, w) d x .
$$

Note that, by Proposition 4.1, such integral is well defined and in fact, if $z, w$ are such that $\operatorname{dist}(z, \partial D), \operatorname{dist}(w, \partial D) \geq R>0$ then we have

$$
|S(z, w)| \leq C R^{2-n}
$$

where $C>0$ only depends on $\lambda$ and $n$. Then, by a well-known identity stemming from (3.3), we have

$$
\left\langle\left(\Lambda_{\gamma_{1}}^{\partial \widetilde{D}}-\Lambda_{\gamma_{2}}^{\partial \widetilde{D}}\right) \eta_{1}, \eta_{2}\right\rangle=\int_{\widetilde{D}}\left(\gamma_{1}-\gamma_{2}\right) \nabla v_{1} \cdot \nabla v_{2} d x
$$

consequently, by (4.2), (4.4), (4.5)

$$
\begin{aligned}
\left\langle\left(\Lambda_{\gamma_{1}}^{\partial \widetilde{D}}-\Lambda_{\gamma_{2}}^{\partial \widetilde{D}}\right) \eta_{1}, \eta_{2}\right\rangle=\int_{D}\left(\gamma_{1}-\gamma_{2}\right) \nabla v_{1} \cdot \nabla v_{2} d x= & \\
& =I_{1}-I_{2}-I_{3}+I_{4}
\end{aligned}
$$

where we denote

$$
\begin{aligned}
& I_{1}=\int_{\partial \widetilde{D} \times \partial \widetilde{D}} \gamma_{0}(z) \gamma_{0}(w) \frac{\partial v_{1}}{\partial \nu}(z) \frac{\partial v_{2}}{\partial \nu}(w) S(z, w) d \sigma_{z} \times \sigma_{w}, \\
& I_{2}=\int_{\partial \widetilde{D} \times \partial \widetilde{D}} \gamma_{0}(z) \gamma_{0}(w) \frac{\partial v_{1}}{\partial \nu}(z) v_{2}(w) \frac{\partial}{\partial \nu_{w}} S(z, w) d \sigma_{z} \times \sigma_{w}, \\
& I_{3}=\int_{\partial \widetilde{D} \times \partial \widetilde{D}} \gamma_{0}(z) \gamma_{0}(w) v_{1}(z) \frac{\partial v_{2}}{\partial \nu}(w) \frac{\partial}{\partial \nu_{z}} S(z, w) d \sigma_{z} \times \sigma_{w}, \\
& I_{4}=\int_{\partial \widetilde{D} \times \partial \widetilde{D}} \gamma_{0}(z) \gamma_{0}(w) v_{1}(z) v_{2}(w) \frac{\partial}{\partial \nu_{z}} \frac{\partial}{\partial \nu_{w}} S(z, w) d \sigma_{z} \times \sigma_{w} .
\end{aligned}
$$


For $z, w \in \mathcal{A}$, the Green's functions $G_{1}(\cdot, z), G_{2}(\cdot, w)$ have no singularity in $\Omega$ and also $\left.G_{1}(\cdot, z)\right|_{\partial \Omega},\left.G_{2}(\cdot, w)\right|_{\partial \Omega} \in H_{c o}^{1 / 2}(\Sigma)$. More specifically, if $z, w \in B_{\rho_{1}}(Q)$, then, by (4.3),

$$
\left\|G_{1}(\cdot, z)\right\|_{H_{c o}^{1 / 2}(\Sigma)},\left\|G_{2}(\cdot, w)\right\|_{H_{c o}^{1 / 2}(\Sigma)} \leq C
$$

where $C$ only depends on $\lambda, \rho_{0}, M_{0}, d_{0}$ and $n$. Thus, recalling (2.7), for $z, w \in$ $B_{\rho_{1}}(Q), S(z, w)$ can be rewritten as follows

$$
\begin{aligned}
S(z, w) & =\int_{D}\left(\gamma_{1}(x)-\gamma_{2}(x)\right) \nabla_{x} G_{1}(x, z) \cdot \nabla_{x} G_{2}(x, w) d x= \\
& =\int_{\Omega}\left(\gamma_{1}(x)-\gamma_{2}(x)\right) \nabla_{x} G_{1}(x, z) \cdot \nabla_{x} G_{2}(x, w) d x= \\
& =\left\langle\left(\Lambda_{\gamma_{1}}^{\Sigma}-\Lambda_{\gamma_{2}}^{\Sigma}\right) G_{1}(\cdot, z), G_{2}(\cdot, w)\right\rangle .
\end{aligned}
$$

Hence,

$$
|S(z, w)| \leq C \varepsilon \text { for every } z, w \in B_{\rho_{1}}(Q)
$$

where we denote

$$
\varepsilon=\left\|\Lambda_{\gamma_{1}}^{\Sigma}-\Lambda_{\gamma_{2}}^{\Sigma}\right\|_{\mathcal{L}\left(H_{c o}^{1 / 2}(\Sigma), H_{c o}^{-1 / 2}(\Sigma)\right)} .
$$

On the other hand, recalling (4.6), the following bound holds

$$
|S(z, w)| \leq C \text { for every } z, w \in \widetilde{\Omega} \backslash \overline{D^{\prime}}
$$

where $C>0$ only depends on $\lambda, \rho_{0}, M_{0}, d_{0}$ and $n$. Moreover, we have the following.

Proposition 4.2 For every $w \in \widetilde{\Omega} \backslash \bar{D}$, the functions $S(\cdot, w), \frac{\partial}{\partial w_{i}} S(\cdot, w), i=1, \ldots, n$ are weak solutions to the elliptic equation

$$
\operatorname{div}\left(\gamma_{0} \nabla v\right)=0 \text { in } \widetilde{\Omega} \backslash \bar{D},
$$

likewise, for every $z \in \widetilde{\Omega} \backslash \bar{D}$, the functions $S(z, \cdot), \frac{\partial}{\partial z_{i}} S(z, \cdot), i=1, \ldots, n$ are weak solutions to the same equation.

Proof. It suffices to verify the weak formulation of (4.16) with an arbitrary test function $\psi \in C_{0}^{\infty}(\widetilde{\Omega} \backslash \bar{D})$. This follows in a straightforward fashion, by repeated use of differentiation under the integral and of Fubini's theorem. Note that use is made of the assumption (4.2).

Using the fact that $S$ solves an elliptic equation in each variable, we can combine the smallness estimate (4.13) with the global bound (4.15), so to estimate the smallness of $S$ and its derivatives on $\partial \widetilde{D} \times \partial \widetilde{D}$. This task can be achieved by an estimate of propagation of smallness, for a general discussion on this concept we refer to [4]. Let us fix $h_{1}<\rho_{2} / 2$ only depending on $\rho_{0}, M_{0}$ such that $\left(\widetilde{\Omega} \backslash \overline{D^{\prime}}\right)_{h_{1}}$ is connected. 
Proposition 4.3 If $v$ is a weak solution to (4.16) then

$$
\|v\|_{L^{2}\left(\left(\widetilde{\Omega} \backslash \overline{D^{\prime}}\right)_{h_{1}}\right)} \leq C\|v\|_{L^{2}\left(B_{\rho_{1}}(Q)\right)}^{\eta}\|v\|_{L^{2}\left(\widetilde{\Omega} \backslash \overline{D^{\prime}}\right)}^{1-\eta}
$$

where $C>0, \eta \in(0,1)$ only depend on the a-priori data $\lambda, E, \rho_{0}, M_{0}, d_{0}, \operatorname{diam}(\Omega)$ and on $n$.

Proof. We refer to [4, Theorem 5.1], of which this proposition is just a special case.

Applying Proposition 4.3 to $v=S(\cdot, w)$, for any $w \in B_{\rho_{1}}(Q)$ we obtain

$$
\|S(\cdot, w)\|_{L^{2}\left(\left(\widetilde{\Omega} \backslash \overline{D^{\prime}}\right)_{h_{1}}\right)} \leq C \varepsilon^{\eta} \text { for every } w \in B_{\rho_{1}}(Q)
$$

and by a further application of Proposition 4.3, with respect to the $w$ variable, we have

$$
\|S(\cdot, \cdot)\|_{L^{2}\left(\left(\widetilde{\Omega} \backslash \overline{D^{\prime}}\right)_{h_{1}} \times\left(\widetilde{\Omega} \backslash \overline{D^{\prime}}\right)_{h_{1}}\right)} \leq C \varepsilon^{\eta^{2}} .
$$

Using the elliptic equation for $S(\cdot, w)$ and the fact that $\partial \widetilde{D}$ is contained in $\left(\widetilde{\Omega} \backslash \overline{D^{\prime}}\right)_{h_{1}}$ and at a distance greater than $\rho_{2} / 2$ from $\partial\left(\widetilde{\Omega} \backslash \overline{D^{\prime}}\right)_{h_{1}}$, by standard interior regularity estimates [10, Theorems 8.24, 8.32] we deduce

$$
\|S(z, \cdot)\|_{L^{2}\left(\left(\widetilde{\Omega} \backslash \overline{D^{\prime}}\right)_{h_{1}}\right)}+\left\|\nabla_{z} S(z, \cdot)\right\|_{L^{2}\left(\left(\widetilde{\Omega} \backslash \overline{D^{\prime}}\right)_{h_{1}}\right)} \leq C \varepsilon^{\eta^{2}} \text { for every } z \in \partial \widetilde{D},
$$

using now the equation for $S(z, \cdot)$ and its first order $z$-derivatives, the interior regularity estimates give

$$
\begin{aligned}
\|S\|_{L^{\infty}(\partial \widetilde{D} \times \partial \widetilde{D})}+\left\|\nabla_{z} S\right\|_{L^{\infty}(\partial \widetilde{D} \times \partial \widetilde{D})} & \\
\left\|\nabla_{w} S\right\|_{L^{\infty}(\partial \widetilde{D} \times \partial \widetilde{D})}+\left\|\nabla_{z} \nabla_{w} S\right\|_{L^{\infty}(\partial \widetilde{D} \times \partial \widetilde{D})} \leq & \leq C \varepsilon^{\beta}
\end{aligned}
$$

where we denote $\beta=\eta^{2}$. Let us now combine the above bounds with (4.8)-(4.12). For instance, we write

$$
I_{1}=\left\langle\Lambda_{\gamma_{1}}^{\partial \widetilde{D}} \eta_{1}, g\right\rangle
$$

where

$$
g(z)=\left\langle\Lambda_{\gamma_{2}}^{\partial \widetilde{D}} \eta_{2}, S(z, \cdot)\right\rangle
$$

hence, with some crude majorization,

$$
\begin{aligned}
&\left|I_{1}\right| \leq C\left\|\eta_{1}\right\|_{H^{1 / 2}(\partial \widetilde{D})}\|g\|_{H^{1 / 2}(\partial \widetilde{D})} \leq \\
& \leq C\left\|\eta_{1}\right\|_{H^{1 / 2}(\partial \widetilde{D})}\left(\|g\|_{L^{\infty}(\partial \widetilde{D})}+\|\nabla g\|_{L^{\infty}(\partial \widetilde{D})}\right) .
\end{aligned}
$$


By the same reasoning and by (4.21) we also have

$$
\left(\|g\|_{L^{\infty}(\partial \widetilde{D})}+\|\nabla g\|_{L^{\infty}(\partial \widetilde{D})}\right) \leq C\left\|\eta_{2}\right\|_{H^{1 / 2}(\partial \widetilde{D})} \varepsilon^{\beta},
$$

and consequently

$$
\left|I_{1}\right| \leq C \varepsilon^{\beta}\left\|\eta_{1}\right\|_{H^{1 / 2}(\partial \widetilde{D})}\left\|\eta_{2}\right\|_{H^{1 / 2}(\partial \widetilde{D})} .
$$

Using a similar approach for the terms in (4.10)-(4.12) we arrive at

$$
\left|\left\langle\left(\Lambda_{\gamma_{1}}^{\partial \widetilde{D}}-\Lambda_{\gamma_{2}}^{\partial \widetilde{D}}\right) \eta_{1}, \eta_{2}\right\rangle\right| \leq C \varepsilon^{\beta}\left\|\eta_{1}\right\|_{H^{1 / 2}(\partial \widetilde{D})}\left\|\eta_{2}\right\|_{H^{1 / 2}(\partial \widetilde{D})},
$$

for any Dirichlet data $\eta_{i} \in C^{1, \alpha}(\partial \widetilde{D}), i=1,2$, and being $C^{1, \alpha}(\partial \widetilde{D})$ dense into $H^{1 / 2}(\partial \widetilde{D})$, the proof of (3.4) and of Theorem 3.1 is complete.

\section{References}

[1] G. Alessandrini, Singular solutions of elliptic equations and the determination of conductivity by boundary measurements, J. Differential Equations 84 (2) (1990), 252-272.

[2] G. Alessandrini, Determining conductivity by boundary measurements, the stability issue. In: Applied and Industrial Mathematics, R. Spigler(ed.), Kluwer, 1991, 317-324.

[3] G. Alessandrini, M. Di Cristo, Stable determination of an inclusion by boundary measurements, SIAM J. Math. Anal. 37 (1) (2005) 200-217.

[4] G. Alessandrini, L. Rondi, E. Rosset, S. Vessella, The stability for the Cauchy problem for elliptic equations, Inverse Problems 25123004 (2009), (47pp).

[5] G. Alessandrini and S. Vessella, Lipschitz stability for the inverse conductivity problem, Adv. Appl. Math., Vol. 35 (2005), 207-241.

[6] H. Ammari and G. Uhlmann, Reconstruction of the potential from partial Cauchy data for Schrödinger equation, Indiana Univ. Math. J. 53 (2004), no.1, 169-183.

[7] A. Bukhgeim, G.Uhlmann, Recovering a potential from partial Cauchy data, Comm. Partial Differential Equations, 27 (2002), 653-668.

[8] M. Di Cristo, Stable determination of an inhomogeneous inclusion by local boundary measurements, J. Comp. Appl. Math. 198 (2007), no. 2 , 414-425.

[9] I. K. Fathallah, Stability for the inverse potential problem by the local Dirichletto-Neumann map for the Schrödinger equation, Appl. Anal. 86 (2007), no. 7, 899-914. 
[10] D. Gilbarg and N. S. Trudinger, Elliptic partial differential equations of second order, volume 224 of Grundlehren der Mathematischen Wissenschaften. Springer-Verlag, Berlin, second edition, 1983.

[11] H. Heck, J.-N. Wang, Stability estimates for the inverse boundary value problem by partial Cauchy data, Inverse Problems, Vol 22 (2006), 1787-1796.

[12] H. Heck, J.-N. Wang, Optimal stability estimate of the inverse boundary value problem by partial measurements, http://arxiv.org/abs/0708.3289.

[13] C. Kenig, J. Sjöstrand, G. Uhlmann, The Calderón problem with partial data, Ann. of Math. (2) 165 (2007), no. 2, 567-591.

[14] O. Imanuvilov, G. Uhlmann, M. Yamamoto, The Calderón problem with partial data in two dimensions, Journal American Math. Society, 23(2010), 655-691.

[15] V. Isakov, On uniqueness in the inverse conductivity problem with local data, Inverse Problems and Imaging, 1 (2007), 95105.

[16] M. Lassas, M. Cheney and G. Uhlmann, Uniqueness for a wave propagation inverse problem in a half space, Inverse Problem 14, no. 3 (1998), 679-684.

[17] W. Littman, G. Stampacchia and H. F. Weinberger, Regular points for elliptic equations with discontinuous coefficients, Ann. Scuola Norm. Sup. Pisa (3) 17 (1963) 43-77.

[18] N. Mandache, Exponential instability in an inverse problem for the Schrödinger equation, Inverse Problems 17 (2001), no. 5, 1435-1444. 